\title{
All-Optical Differential Current Detection Technique for Unit Protection Applications
}

\author{
Grzegorz Fusiek, Philip Orr, Hao Wang, Paweł Niewczas \\ Institute for Energy and Environment, \\ Department of Electronic and Electrical Engineering, University of Strathclyde \\ Glasgow, UK \\ gfusiek@eee.strath.ac.uk
}

\begin{abstract}
In this paper we demonstrate a novel, all-optical differential current protection scheme. By monitoring the optical power reflected from two matched hybrid fiber Bragg grating current sensors and using a simple optoelectronic threshold detector, an immediate response to an increase in differential current is achieved. A preliminary laboratory embodiment is constructed in order to characterize the performance of the scheme. The proposed technique does not require a complex sensor interrogation scheme, usually characterized by a limited sampling frequency, and thus will be capable of facilitating inexpensive and fast-acting differential protection over long distances.
\end{abstract}

Keywords - fiber Bragg grating, piezoelectric transducer, voltage sensor, current sensor, optical, differential protection

\section{INTRODUCTION}

Differential unit protection is widely used for transmission lines, transformers, tapped lines or busbars in power systems. In general, it relies on the measurement and comparison of currents at two extreme locations of a protected zone [1]. In normal (no fault) conditions the input and output currents of the protected zone are equal. Any fault within the zone produces a difference - in magnitude, phase or both - between the measured currents. As the protected zones often span tens of $\mathrm{km}$ and upwards, the use of high-speed communication and sampling synchronization is required for microprocessor-based differential relays, and attempts to improve and simplify their operation, for example by employing GPS (Global Positioning System) synchronization, have been made [2].

As an alternative to conventional protection systems, we have previously proposed the use of optical voltage and current sensors for different protection applications, including differential and distance protections [3]-[5]. It was demonstrated that an optical sensor system could replace a conventional system or could be used to transmit measurement data or tripping signals to protection relays without the complexity and bottlenecks associated with conventional relays' communication and synchronization techniques [5].

In this paper, we propose a novel all-optical protection scheme that can be used in applications where detailed information about individual measured currents is not required and detection of the differential current is sufficient. In such cases, the proposed all-optical solution significantly reduces the cost of the protection system as no interrogator is required. In addition, the speed of operation of an associated relay can be extremely fast due to the all-optical nature of the differential calculation, and the simplicity of the optoelectronic threshold.

\section{MEASUREMENT SYSTEM}

\section{A. Optical voltage and current sensors}

In order to monitor current at two ends of the protected zone, we employ previously developed hybrid fiber-optic voltage and current sensors [6], [7]. The optical voltage sensor utilizes a piezoelectric stack (transducer) which expands or contracts proportionally to a voltage applied to its external electrodes. The strain generated by the voltage in the transducer is monitored by a fiber Bragg grating (FBG) sensor bonded to the stack as shown in Fig. 1 (a). The strain exerted on the FBG produces a corresponding shift in its peak reflected wavelength. Thus, the peak wavelength shift can be calibrated in terms of voltage.
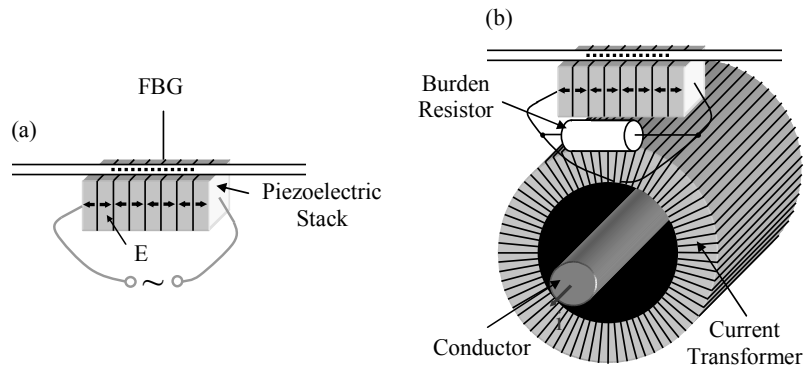

Fig. 1. Optical (a) voltage and (b) current sensors.

The optical current sensor, shown in Fig. 1 (b), utilizes a small ferrite core current transformer (CT), a burden resistor for current to voltage conversion and a hybrid piezoelectric voltage sensor as described above. The measured primary current is transferred to a smaller secondary current flowing through the resistor and a voltage developed across its terminals is monitored by the voltage sensor. Thus, the FBG wavelength shift may be related to current.

\section{B. Optical fault detection concept}

The novel concept of all-optical fault detection relies on the use of a pair of matched current sensors utilizing two identical FBG sensors attached to piezoelectric transducers to monitor 
currents at the two extreme locations of the protected zone (see Fig. 2).

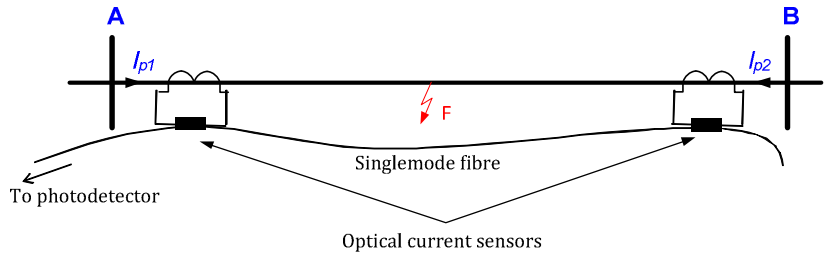

Fig. 2. Fault detection scheme.

In power systems, both phase-phase and phase-earth faults occurring either inside the protected zone (internal faults) or outside the protected zone (external faults) alter current within the zone. Under fault conditions, currents entering and exiting the zone differ in magnitude and/or phase (direction).

As shown in Fig. 3 (a), when two gratings having similar spectral profile (i.e. peak wavelength, reflection and bandwidth) are illuminated by a light source, they reflect a certain amount of spectrum, and the reflected optical power can be detected by a photodetector. When the FBGs are spaced spectrally apart, the reflected power distribution between the sensors is changed, as shown in Fig 3 (b). The amount of reflected power will depend on the separation of FBG spectra and the degree in which they overlap because the total power incident on the photodetector is proportional to the area under the curves shown in Fig. 3.
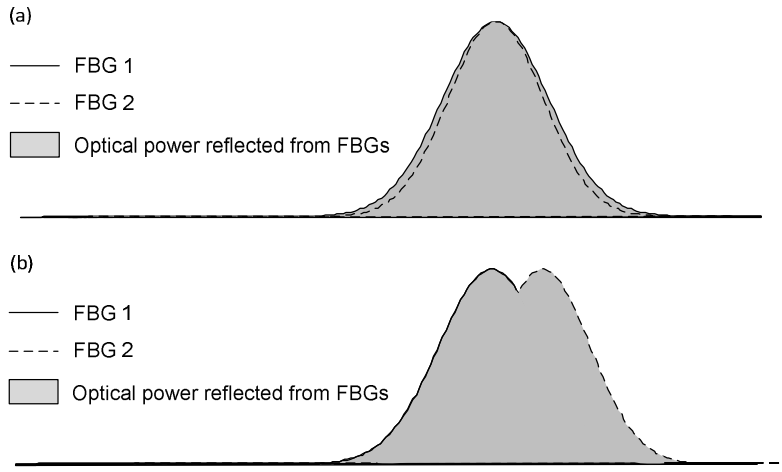

Fig. 3. Optical power distribution between two FBGs: (a) nominal condition - FBGs are aligned, (b) fault condition - FBGs are spaced apart.

Under nominal (no fault) conditions, both current sensors measure equal ac currents and the reflected sensor wavelengths shift identically - provided that the sensors are kept at the same temperature. The power recorded by the photodetector in this case would be constant, since the integrated reflected spectrum is constant while currents match. Under fault conditions, the sensors would measure currents having different amplitudes or phases, and hence the total reflected power is modulated. This translates to a modulation of the photodetector output voltage which, after thresholding, may be used for fault indication. Thus, by using two identical current sensors placed at the boundaries of the protected zone, a sensor for differential current is produced, which may be utilized as a fast-acting and lightweight all-optical differential protection scheme.

\section{SOFTWARE SIMULATION}

To theoretically evaluate the proposed method of alloptical fault detection, a simulation program was written in LabVIEW (National Instruments). The FBG spectral profiles were approximated by normalized Gaussian functions.

Assuming that two FBGs have normalized Gaussian profiles and the light source illuminating the sensors has a constant spectral profile, the optical power reflected from the FBGs and detected by a photodetector is given by the integral of the FBG reflection functions according to the following equation:

$$
\mathrm{P}_{\text {det }}(\lambda)=\int F B G(\lambda) d \lambda
$$

where $F B G(\lambda)$ is the total reflection spectrum given by

$$
F B G(\lambda)=F B G 1(\lambda)+(1-F B G 1(\lambda)) \cdot F B G 2(\lambda)
$$

and $F B G 1(\lambda)$ and $F B G 2(\lambda)$ are the FBGs reflection spectra approximated by

$$
\begin{aligned}
& F B G 1(\lambda)=\exp \left[\frac{-\left(\lambda-\lambda_{F B G 1}\right)^{2}}{2 \sigma_{F B G 1}^{2}}\right] \\
& F B G 2(\lambda)=\exp \left[\frac{-\left(\lambda-\lambda_{F B G 2}\right)^{2}}{2 \sigma_{F B G 2}^{2}}\right]
\end{aligned}
$$

In the above equations, $\lambda_{F B G}$ is the FBG peak wavelength and $\sigma_{F B G}$ is a parameter determining the FBG bandwidth. The program allowed for sweeping the simulated FBG spectra over the required wavelength range, and the calculated total power levels were recorded in a data file. From a number of simulations performed, only representative examples are presented below.

Fig. 4 shows the total optical power reflected from the sensors as a function of the spacing between them. The greater the difference between the FBG bandwidths, the smaller the changes in optical power that can be observed. Unequal FBG maximum reflections affect the total power less than unequal bandwidths. Note that in all cases the reflected power was normalized in these simulations.

Fig. 5 shows the results of simulation when both sensors are subject to sinusoidal FBG peak shifts having the same amplitude and phase. This scenario corresponds to nominal (no fault) conditions in the power system and the sensors measure equal currents. As can be seen, the optical power level is constant.

Fig. 6 depicts the case when both sensors are subjected to sinusoidal FBG peak shifts having the same amplitude and inverted phases. This scenario could be produced in power networks with generation at each end of the faulted zone, where fault inception would result in a reversal of power flow at one end (towards the fault). In this case, the optical power is modulated. By detecting changes in the optical power modulation, fault occurrence can be detected and the trip signal generated. 


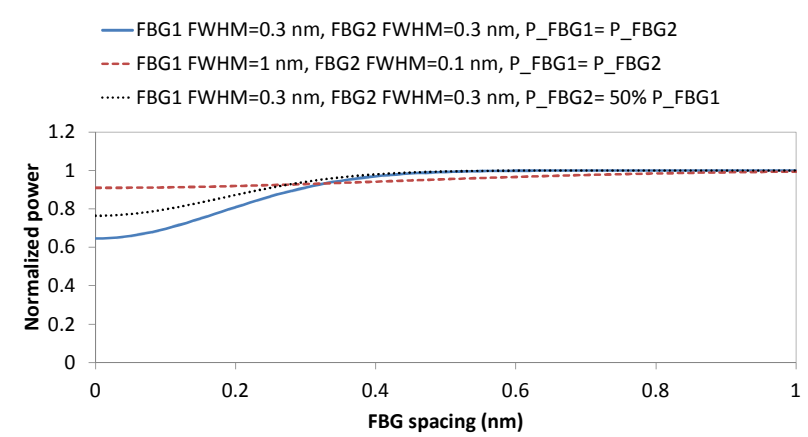

Fig. 4. Normalized optical power levels versus FBG spacing for two FBGs with different (or equal) bandwidths and reflections.

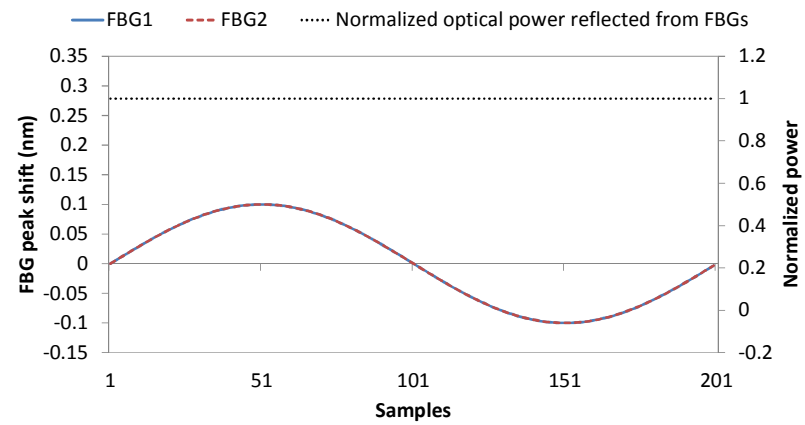

Fig. 5. Normalized optical power modulation (top trace) during the sinusoidal oscillation of FBG peaks (bottom traces). FBGs are subjected to sine waves having the same amplitudes and phases. The waveforms are offset for clarity.

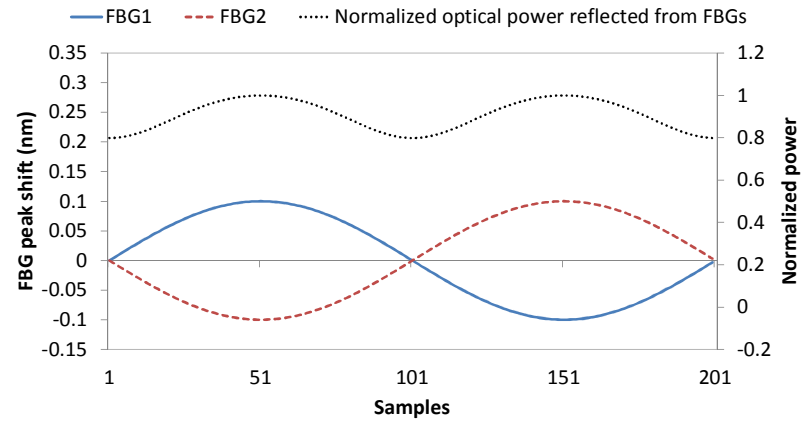

Fig. 6. Normalized optical power modulation (top trace) during the sinusoidal oscillation of FBG peaks (bottom traces). FBGs are subjected to sine waves having the same amplitudes and inverted phases. The waveforms are offset for clarity.

The depth of power modulation will depend on the sensor sensitivity to voltage/current. By careful sensor design, the FBG shift can be tailored so that the required depth of optical power modulation can be achieved at the maximum current/voltage level.

\section{EXPERIMENTAL EVALUATION}

\section{A. Distance protection scheme configuration}

In order to demonstrate the proposed technique, the experimental setup shown in Fig. 7 was constructed. Two FBG-based sensors were illuminated by a broadband superfluorescent source having central wavelength at $1550 \mathrm{~nm}$ and 40-nm bandwidth. The optical signals reflected from the sensors having bandwidths of $0.2 \mathrm{~nm}$ and peaks at $1549.69 \mathrm{~nm}$ and $1549.74 \mathrm{~nm}$ were monitored and converted to the electrical representation by means of a photodetector. A 16-bit PXI data acquisition card and a PXI controller (both from National Instruments) were used to acquire signals from the photodetector which were then processed in a PC. The data acquisition sampling rate was set at $10 \mathrm{kS} / \mathrm{s}$.

An amplifier module (APTS3AI, Relay Engineering Services Ltd) was used to generate voltage waveforms modeled in the PC and generated by the PXI unit.

As current readings are achieved through voltage measurements across the burden resistor of the current sensor, and the relation between voltage and current is linear, voltage sensors were used instead of current sensors to simplify the experimental circuitry. This simplification proves the concept of the proposed all-optical fault detection and allows for direct connection of the PXI unit to the APTS3AI module and reduction of the required laboratory equipment.

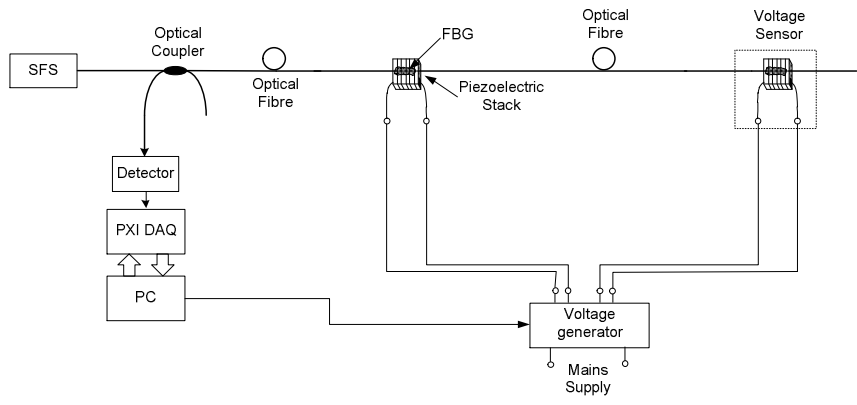

Fig. 7. Experimental setup.

\section{B. Fault scenarios and experimental results}

A number of different fault scenarios were investigated, of which only three representative cases are presented below. As summarized in TABLE I. during the pre-fault condition both sensors were subjected to voltages of the same amplitude and phase. During the fault condition, they were subjected to voltages of different amplitude and/or phase. The experiments were carried out at room temperature.

TABLE I. Pre-Fault and Fault Conditions

\begin{tabular}{|l|c|c|c|c|c|c|}
\hline \multirow{2}{*}{ Condition } & \multicolumn{3}{|c|}{ Pre-Fault } & \multicolumn{3}{c|}{ Fault } \\
\cline { 2 - 7 } & FBG1 & FBG2 & Phase & FBG1 & FBG2 & Phase \\
\hline Fault A & $100 \mathrm{~V}$ & $100 \mathrm{~V}$ & $0^{\circ}$ & $0 \mathrm{~V}$ & $300 \mathrm{~V}$ & $0^{\circ}$ \\
\hline Fault B & $200 \mathrm{~V}$ & $200 \mathrm{~V}$ & $0^{\circ}$ & $200 \mathrm{~V}$ & $200 \mathrm{~V}$ & $180^{\circ}$ \\
\hline Fault C & $200 \mathrm{~V}$ & $200 \mathrm{~V}$ & $0^{\circ}$ & $400 \mathrm{~V}$ & $300 \mathrm{~V}$ & $180^{\circ}$ \\
\hline
\end{tabular}

Fig. 8 shows the results of the representative fault detections. In the figure, the bottom traces represent $50-\mathrm{Hz}$ voltage waveforms modeled in a PC and captured immediately prior to generation by the PXI unit. These signals are amplified and generated by the APTS unit before they can be measured by the optical sensors.

Fig. 9 depicts a trip signal generated in response to the Fault $\mathrm{C}$ scenario. The photodetector output modulation was 
monitored by tracing peak-to-peak values of the signal and compared to a set threshold. Peak-to-peak values were estimated from the data length equal to one period of the voltage waveform.
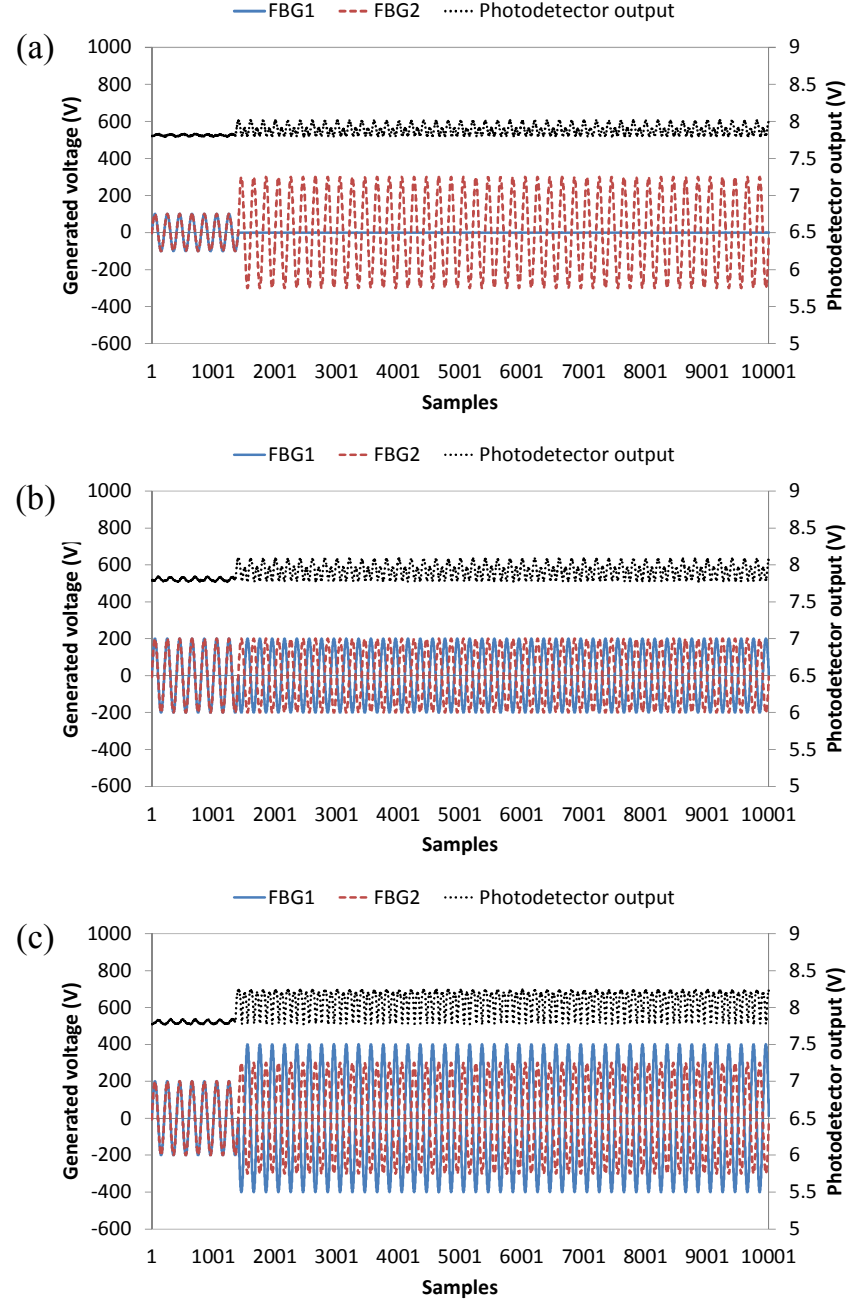

Fig. 8. Photodetector output (top trace) and voltage on the sensors bottom traces) during (a) Fault A, (b) Fault B and (c) Fault C scenarios. The waveforms are offset for easier comparison.

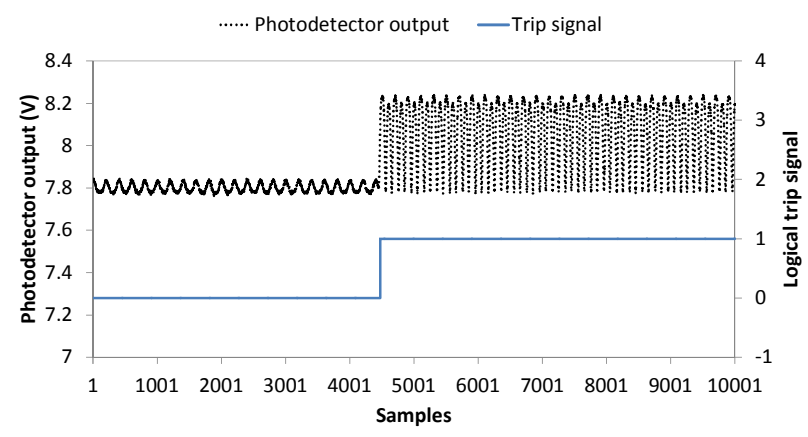

Fig. 9. Trip signal generation in response to Fault $\mathrm{C}$ detection.

\section{CONCLUSIONS}

In this paper, we have proposed and successfully implemented a novel approach to differential unit protection using two identical optical current sensors. Since the novel approach does not require the use of interrogators, the cost of the measurement system is significantly reduced. By monitoring the reflected optical power modulation and comparing it to a set threshold, fault detection has been demonstrated. Three different fault scenarios evaluated in the laboratory have shown that the new technique has the potential to enable very fast-acting and inexpensive all-optical unit protection.

It should be noted that all the results presented in the paper were achieved while sensors were kept at the same temperature. In a practical deployment, the sensors may be at different temperatures due, for example, to their distant locations along a transmission line. The FBG peak shifts due to temperature may then introduce errors in fault detection and false tripping. Future work will address these temperaturerelated issues and suitable temperature compensation methods will be developed to ensure reliable fault detection over varying environmental conditions with a minimum of fault positives.

\section{REFERENCES}

[1] AREVA T\&D, Network Protection and Automation Guide. $1^{\text {st }}$ Ed., Cayfosa, Barcelona, ISBN 2-9518589-0-6, July 2002

[2] I. Hall, P. G. Beaumont, G. P. Baber, I. Shuto, M. Saga, K. Okuno, H. Ito, "New Line Current Differential Relay using GPS Synchronization", 2003 IEEE Bologna Power Tech Conference, June 23-26, Bologna, Italy

[3] P. Orr, P. Niewczas, A. Dysko, C. Booth, "FBG-based fiberoptic current sensors for power systems protection: Laboratory evaluation," Universities Power Engineering Conference (UPEC), 2009 Proceedings of the 44th International , pp.1-5, 1-4 Sept. 2009

[4] P. Orr, G. Fusiek, P. Niewczas, A. Dysko, C. Booth, F. Kawano, G. Baber, "Distributed optical distance protection using FBGbased voltage and current transducers," IEEE Power and Energy Society General Meeting, pp. 1-5, 24-29 July 2011

[5] P. Orr, G. Fusiek, C.D. Booth, P. Niewczas, A. Dyśko, F. Kawano, P. Beaumont, T. Nishida, 'Flexible protection architectures using distributed optical sensors', IET Conf. Pub. 2012, 155 (2012)

[6] L. Dziuda, P. Niewczas, G. Fusiek, J. R. McDonald, "Hybrid Fiber-Optic Voltage Sensor for Remote Monitoring of Electrical submersible Pump Motors", Optical Engineering, Vol. 44, No. 6, pp 64401-1-6, June 2005

[7] L. Dziuda, G. Fusiek, P. Niewczas, G. Burt, and J.R. McDonald, "Laboratory Evaluation of the Hybrid Fiber-Optic Current Sensor", Sensors and Actuators, A: Physical, Vol. 136, No. 1, pp. 184-190, May 1, 2007 